\title{
MÉTODOS DE DETERMINAÇÃO DA DENSIDADE DO SOLO EM DIFERENTES SISTEMAS DE MANEJO
}

\author{
Fernanda Coelho Gonçalves ${ }^{1}$, Indiamara Marasca², Saulo Fernando Gomes De Souza ${ }^{3}$, \\ Leandro Augusto Felix Tavares ${ }^{4} \&$ Paulo Roberto Arbex Silva ${ }^{5}$
}

\begin{abstract}
RESUMO: A densidade do solo é um atributo muito utilizado para caracterizar fisicamente a estrutura do solo, constituindo um indicador de sua compactação. O objetivo deste trabalho foi comparar os valores de densidade do solo medidos pelo método do torrão parafinado e pelo método do anel volumétrico em sistema convencional, plantio direto e cultivo mínimo em um Nitossolo Vermelho distroférrico, textura argilosa, na região de Botucatu, SP. O experimento foi instalado em esquema fatorial $3 \times 2$, com delineamento em blocos casualizados, com quatro repetições. Foram determinados os valores de densidade do solo pelo método do torrão parafinado e pelo método do anel volumétrico para os sistemas de manejo estudados. Os valores de densidade obtidos pelo método do torrão parafinado foram estatisticamente superiores aos obtidos pelo método do anel volumétrico. Não houve diferença entre os manejos quando comparados os valores de densidade do solo obtidos pelo método do torrão parafinado. O solo sob manejo convencional apresentou menor densidade quanto comparados os valores de densidade do solo obtidos pelo método do anel volumétrico. O método do anel volumétrico foi mais sensível que o método do torrão parafinado para mostrar diferenças entre sistemas de manejo.
\end{abstract}

PALAVRAS-CHAVE: propriedades físicas, anel volumétrico e torrão parafinado.

\section{METHODS FOR DETERMINING THE SOIL BULK DENSITY UNDER DIFFERENT MANAGEMENT SYSTEMS}

ABSTRACT: Soil bulk density is an attribute often used to characterize soil physical structure, being an indicator of soil compaction. The objective of this study was to compare the values of bulk density measured by the paraffin sealed clod and volumetric ring methods in conventional, no-tillage and minimum tillage systems on a Dystroferric Red Nitosol, clayey, in Botucatu, SP. The experiment design was a 3x2 factorial arrangement in randomized block with four replications. The density values obtained by the paraffin sealed clod method were statistically higher than those obtained by the volumetric ring method. There was no difference between the managements when comparing the values of soil density obtained by the paraffin sealed clod method. The soil under conventional management showed lower density when comparing the values of soil density obtained by the volumetric ring. The volumetric ring method was more sensitive to show differences between the management systems than the paraffin sealed clod method.

KEYWORDS: physical properties, volumetric ring and paraffin sealed clod.

\footnotetext{
${ }^{1}$ FCA/UNESP - Botucatu. Email: fecoelhog@yahoo.com.br

${ }^{2}$ FCA/UNESP - Botucatu. Email: marasca@ fca.unesp.br

${ }^{3}$ FCA/UNESP - Botucatu. Email: saulo@fca.unesp.br

4 FCA/UNESP - Botucatu. Email: leandrotavares@fca.unesp.br

5 FCA/UNESP - Botucatu. Email: arbex@fca.unesp.br
} 


\section{INTRODUÇÃO}

A deterioração da qualidade do solo tem sido amplamente estudada e debatida pela ciência do solo, devido principalmente, a retirada da cobertura e/ou uso intensivo da mecanização agrícola nas operações de preparo e cultivo do solo (ASSIS; LANÇAS, 2005).

Os sistemas de preparo do solo promovem modificações nas propriedades físicas, como a densidade e a porosidade (TORMENA et al., 2002). A avaliação das propriedades físicas sob diferentes sistemas de preparo do solo é importante para caracterizar o ambiente físico para o crescimento radicular (TAVARES FILHO et al., 2001; CAVALIERI et al., 2006).

Entre as propriedades físicas mais avaliadas está a densidade, que fornece indicações a respeito do estado de compactação do solo (REICHARDT; TIMM, 2008), podendo indicar a necessidade de realização de prática mecânica de descompactação do solo com a utilização do subsolador ou do escarificador.

O método do anel volumétrico (MAV) é considerado como método padrão de amostragem para a avaliação da densidade do solo, o qual consiste na amostragem do solo com estrutura indeformada num anel (cilindro metálico) de volume conhecido (EMBRAPA, 1997). Porém, esse método pode provocar uma possível compactação do solo pelo atrito cilindro-solo durante a penetração do mesmo no solo, principalmente quando o solo estiver com umidade acima do ponto de friabilidade, ou de fraturar ou cisalhar o solo quando o mesmo estiver seco (BLAKE; HARTG, 1986).

Outro método utilizado para a determinação da densidade do solo é o método do torrão parafinado (MTP), que consiste na coleta de torrões de volume variável, secos ao ar, e impermeabilizado em parafina líquida. O volume dos torrões é determinado pelo volume de água deslocado pelos mesmos quando imersos em água (KIEHL, 1979). Apresenta a desvantagem de poder segregar a amostra por ocasião da coleta dos torrões em solos de textura arenosa que foram mobilizados e não completamente reconsolidados, desconsiderando os macroporos existentes entre os torrões (SILVA et al. 2000). O MTP é bastante útil em solos pedregosos, ou quando o solo estiver muito seco que impeça a penetração do cilindro no solo (KIEHL, 1979).

Os valores de densidade do solo determinados pelo MTP são geralmente maiores que os valores determinados pelo MAV, o que está relacionado com a possibilidade da parafina penetrar em macroporos e fendas durante a impermeabilização do torrão, e também, com a segregação e perda de macroporos no momento da coleta do torrão (BLAKE; HARTGE, 1986; VAN REMORTEL; SHIELDS, 1993; SILVA et. al., 2000; PIRES et al., 2011). Contudo, há necessidade de maior investigação das diferenças e do comportamento desses dois métodos para diferentes sistemas de manejo do solo.
O objetivo deste trabalho foi comparar os valores de densidade do solo medidos pelo MTP e MAV em diferentes sistemas de manejo em Nitossolo Vermelho distroférrico.

\section{MATERIAL E MÉTODOS}

O experimento foi conduzido no ano agrícola 2009/2010, na Fazenda Experimental Lageado, pertencente à Faculdade de Ciências Agronômicas - UNESP, localizada no município de Botucatu - SP, na região centro oeste do Estado de São Paulo, tendo como coordenadas geográficas aproximadas de Latitude $22^{\circ}$ $51^{\prime} \mathrm{S}$ e Longitude $48^{\circ} 26^{\prime} \mathrm{W}$ de Greenwich, altitude média de 770 metros, clima subtropical chuvoso, apresentando inverno seco, tipo $\mathrm{Cfa}$, de acordo com o critério de Köeppen. O solo da área experimental foi classificado como Nitossolo Vermelho distroférrico textura argilosa, de acordo com os critérios do Sistema Brasileiro de Classificação de Solos (EMBRAPA, 2006). A declividade da área é de $8 \%$.

O experimento foi instalado em esquema fatorial $3 \times 2$, sendo três sistemas de manejo (convencional, plantio direto e cultivo mínimo) e dois métodos de determinação da densidade do solo (torrão parafinado e anel volumétrico), utilizando o delineamento em blocos casualizados, com quatro repetições, em parcelas de $15 \mathrm{~m}$ de comprimento e 7,5m de largura.

A área experimental vinha sendo cultivada em sistema plantio direto há pelo menos 11 anos. Desde então sempre fazendo a rotação de culturas com soja (Glycine max L.) e milho (Zea mays L.) nas safras de verão e triticale (Triticum turgidocereale), aveia preta (Avena strigosa Schreb) e crambe (Crambe abyssinica Hochst) como culturas de inverno. Em dezembro de 2009 a área recebeu os preparos de solo e foi cultivada com a cultura do milho. No momento da realização dos preparos e semeadura do milho a área estava com baixa quantidade de resíduos vegetais, sendo uma pequena porcentagem desta pertencente à cultura do crambe, cultivada anteriormente, e a maior parte pertencente às plantas daninhas dessecadas.

Para o preparo convencional foi utilizada uma grade aradora intermediária modelo GAICR com 20 discos recortados em ambas as seções, espaçados em 0,27 m, 28 polegadas de diâmetro, largura de corte de 2,57 m e massa total de $2272 \mathrm{~kg}$.

O cultivo mínimo foi caracterizado pela utilização de um escarificador modelo Jumbo Matic JMAD-7, de arrasto, equipado com sete hastes, espaçadas em $0,4 \mathrm{~m}$, ponteiras de $0,08 \mathrm{~m}$ de largura, conjugado com discos de corte, cilindro destorroador/nivelador na parte traseira do equipamento. Foi utilizada a regulagem para a profundidade de trabalho de $0,20 \mathrm{~m}$.

A semeadura foi realizada com uma semeadoraadubadora de precisão modelo PS-6, de arrasto para plantio direto, com quatro linhas para semeadura de 
milho, espaçadas em $0,85 \mathrm{~m}$, com disco de corte, sulcadores para adubo e sementes tipo discos duplos concêntricos de $331 \mathrm{~mm}$ de diâmetro, dosadores de adubo tipo rotor dentado horizontal e dosadores de sementes tipo discos horizontais perfurados.

A coleta para determinação da densidade do solo foi realizada em abril de 2010, depois da colheita do milho, nas camadas de 0-0,10 m e 10-0,20 m. Foram coletados torrões com aproximadamente $45 \mathrm{~cm}^{3}$ para o MTP e, para o MAV, foram coletadas amostras em cilindros de aço com bordas bisseladas (diâmetro de $4,88 \mathrm{~cm}$, altura de $5,28 \mathrm{~cm}$ e volume de $98,94 \mathrm{~cm}^{3}$ ). As amostras foram coletadas em um ponto central da parcela, na entrelinha da cultura do milho, e após a limpeza da superfície do terreno. Em cada parcela foi coletada uma amostra para cada método de determinação da densidade e para cada profundidade.

Para a obtenção da densidade do solo foram utilizados os métodos do torrão parafinado (MTP), conforme Kiehl (1979) e do anel volumétrico (MAV), segundo Embrapa (1997). Os valores obtidos a partir dessa coleta foram utilizados nas comparações entre os métodos de determinação.

Os resultados foram submetidos à análise de variância, e a comparação entre as médias foi feita pelo teste de Tukey, a 5\% de probabilidade utilizando o Sistema de Análise Estatística - Winstat (MACHADO, 2001).

\section{RESULTADOS E DISCUSSÃO}

A Tabela 1 apresenta a comparação entre os valores médios de densidade do solo, determinados pelos métodos do torrão parafinado e anel volumétrico, para os diferentes manejos. Houve diferença significativa entre os valores médios de densidade do solo obtidos pelo MTP e MAV.

Para o solo sob manejo convencional os valores de densidade determinados pelo MTP foram significativamente maiores que os valores obtidos pelo MAV para as duas camadas analisadas. No solo sob cultivo mínimo os valores de densidade determinados pelo MTP foram significativamente maiores apenas na camada 0-0,10 m, e para o solo sob plantio direto houve diferença apenas na camada $0,10-0,20 \mathrm{~m}$, sendo os valores obtidos pelo MTP superiores aos obtidos pelo MAV.

Os maiores valores de densidade obtidos pelo MTP podem estar associados à penetração da parafina em macroporos e fendas existentes no torrão, ou à segregação no momento da coleta dos torrões em solos que foram mobilizados acarretando maiores valores de densidade, pois desconsidera os macroporos existentes entre os torrões (BLAKE; HARTGE, 1986; SILVA et al., 2000).

Van Remortel e Shields (1993) encontraram valores maiores de densidade do solo para o MTP do que para o MAV. Pires et al. (2011) também observaram diferenças significativas entre os valores de densidade obtidos pelo MAV e pelo MTP em Nitossolo Vermelho eutrófico sob manejo convencional, os valores de densidade pelo MTP foram, em média, $0,15 \mathrm{~kg} \mathrm{dm}^{-3}$ maiores. Neste estudo os valores de densidade foram, em média, $0,12 \mathrm{~kg} \mathrm{dm}^{-3}$ maiores. Silva et al. (2000) compararam os MTP e MAV para amostras de um Argissolo Vermelho distrófico arênico coletadas na superfície do solo e constataram diferenças de $0,14 \quad \mathrm{~kg} \mathrm{dm}^{-3}$ entre essas duas metodologias. Esses autores concluíram que devem ser evitadas as comparações entre sistemas de manejo que utilizam métodos distintos na determinação da densidade do solo.

Tabela 1: Comparação entre os valores médios de densidade do solo ( $\left.\mathrm{kg} d \mathrm{~m}^{-3}\right)$, determinados pelos métodos do torrão parafinado (MTP) e anel volumétrico (MAV), em Nitossolo Vermelho distroférrico sob manejo convencional, cultivo mínimo e plantio direto.

\begin{tabular}{|c|c|c|}
\hline \multirow[b]{2}{*}{ Manejo } & \multicolumn{2}{|c|}{ Métodos } \\
\hline & $\begin{array}{c}\text { Torrão } \\
\text { parafinado }\end{array}$ & Anel volumétrico \\
\hline & \multicolumn{2}{|c|}{ Camada $0-0,10 \mathrm{~m}$} \\
\hline Convencional & 1,31 a $\mathrm{A}$ & $1,09 \mathrm{~b}$ B \\
\hline Plantio Direto & 1,34 a $\mathrm{A}$ & 1,29 a A \\
\hline Cultivo Mínimo & 1,35 a $\mathrm{A}$ & $1,16 \mathrm{~b} \mathrm{AB}$ \\
\hline DMS (métodos) & \multicolumn{2}{|c|}{0,13} \\
\hline DMS (manejo) & \multicolumn{2}{|c|}{0,16} \\
\hline $\mathrm{CV}(\%)$ & \multicolumn{2}{|c|}{6,82} \\
\hline & \multicolumn{2}{|c|}{ Camada $0,10-0,20 \mathrm{~m}$} \\
\hline Convencional & 1,31 a $\mathrm{A}$ & $1,18 \mathrm{~b} \mathrm{~B}$ \\
\hline Plantio Direto & 1,34 a $\mathrm{A}$ & $1,28 \mathrm{~b} \mathrm{~A}$ \\
\hline Cultivo Mínimo & 1,28 a $\mathrm{A}$ & 1,26 a $\mathrm{A}$ \\
\hline DMS (métodos) & \multicolumn{2}{|c|}{0,05} \\
\hline DMS (manejo) & \multicolumn{2}{|c|}{0,06} \\
\hline $\mathrm{CV}(\%)$ & \multicolumn{2}{|c|}{2,56} \\
\hline
\end{tabular}

Médias seguidas de mesma letra minúscula na linha e maiúscula na coluna, não diferem significativamente pelo Teste de Tukey a 5\% de probabilidade.

Não foi verificada diferença significativa entre os manejos quando comparados os valores de densidade obtidos pelo MTP. Já, quando o método utilizado para a determinação da densidade foi o MAV, observou-se diferença significativa entre o manejo convencional e o sistema plantio direto na camada $0-0,10 \mathrm{~m}$, e entre o manejo convencional e os demais sistemas de manejo na camada $0,10-0,20 \mathrm{~m}$.

Os valores de densidade obtidos para o solo sob plantio direto foram maiores que os obtidos para o solo sob manejo convencional nas duas camadas avaliadas. Em muitos estudos são observados maiores valores de densidade do solo sob plantio direto, em relação ao 
preparo convencional, sendo esses relacionados ao adensamento natural devido à ausência de revolvimento, como também, à compactação ocasionada pelo tráfego de máquinas (STONE; SILVEIRA, 2001; FALLEIRO et al., 2003; SILVEIRA et al., 2008).

O solo sob cultivo mínimo apresentou valores de densidade intermediários aos obtidos para o solo sob manejo convencional e sistema plantio direto na camada 0-0,10 m sem, no entanto, diferir estatisticamente desses dois tratamentos. Isso indica que houve diminuição do efeito benéfico da escarificação sobre a densidade do solo desde o preparo até a colheita do milho. Na camada 0,10-0,20 m, a densidade do solo sob cultivo mínimo foi estatisticamente igual à densidade do solo sob plantio direto e maior que a densidade do solo sob manejo convencional, não apresentando mais efeito da escarificação nessa camada após o ciclo da cultura. Esse resultado concorda com Ralisch et al. (2001), que concluíram que a escarificação tem efeito somente para um ciclo de cultura, não tendo efeito residual para outras culturas subseqüentes. Já Nicoloso et al. (2008), relataram que a escarificação reduziu a densidade do solo em 4,8\% em relação ao plantio direto nove meses depois do preparo do solo.

O MTP superestimou os valores de densidade, principalmente no manejo convencional, onde ocorre maior mobilização do solo. Para solos mobilizados, o MAV seria mais indicado por apresentar resultados confiáveis (SILVA et. al., 2000). A utilização do MTP para a determinação da densidade pode levar a uma interpretação errada sobre o estado de compactação do solo, acarretando em utilização desnecessária de prática mecânica de descompactação.

É importante salientar que os valores de densidade obtidos estão abaixo dos níveis considerados críticos para as culturas, que variam de 1,4 a 1,6 kg dm-3 para solos argilosos (REICHERT et al., 2003).

\section{CONCLUSÃO}

Os métodos do torrão parafinado e do anel volumétrico resultaram em valores de densidade do solo diferentes.

Não houve diferença entre os manejos quando comparados os valores de densidade do solo obtidos pelo método do torrão parafinado.

O solo sob manejo convencional apresentou menor densidade quanto comparados os valores de densidade do solo obtidos pelo método do anel volumétrico.

O método do anel volumétrico foi mais sensível que o método do torrão parafinado para mostrar diferenças entre sistemas de manejo.

\section{REFERÊNCIAS}

ASSIS, R. L.; LANÇAS, K. P. Avaliação dos atributos físicos de um Nitossolo Vermelho distroférrico sob sistema plantio direto, preparo convencional e mata nativa. Revista Brasileira de Ciência do Solo, Viçosa, v. 29, n. 4, p. 515-522, 2005. Disponível em: <http://www.scielo.br/pdf/rbcs/v29n4/26100.pdf>. Acesso em: 18 de novembro de 2013.

BLAKE, G. R.; HARTGE, K. H. Bulk density. In: KLUTE, A. (Ed.). Methods of soil analysis: physical and mineralogical methods. Part 1. American Society of Agronomy, 1986. p. 363-375.

CAVALIERI, K. M. V. et al. Efeitos de sistemas de preparo nas propriedades físicas de um Latossolo Vermelho distrófico. Revista Brasileira de Ciência do Solo, Viçosa, v. 30, n. 1, p. 137-47, 2006. Disponível em: <http://www.scielo.br/pdf/rbcs/v30n1/a14v30n1.pd f $>$. Acesso em: 18 de novembro de 2013.

EMBRAPA. Centro Nacional de Pesquisa em Solos. Sistema Brasileiro de Classificação de Solos. Brasília, 2006. 306p.

EMBRAPA. Centro Nacional de Pesquisa de Solos. Manual de métodos de análise de solo. 2. ed. Brasília, 1997. 212p.

FALLEIRO, R. M. et al. Influência dos sistemas de preparo nas propriedades químicas e físicas do solo. Revista Brasileira de Ciência do Solo, Viçosa, v. 27, n. 6, p. 1097-1104, 2003. Disponível em: <http://www.scielo.br/pdf/rbcs/v27n6/19204.pdf>. Acesso em: 18 de novembro de 2013.

KIEHL, E. J. Manual de edafologia: relações solo-planta. São Paulo: Editora Agronômica Ceres, 1979. 264p.

\section{MACHADO, A. A. Sistema de análise estatística para Windows (WINSTAT). Pelotas: Universidade Federal de Pelotas, 2001. 1 CD- ROM.}

NICOLOSO, R. S. et al. Eficiência da escarificação mecânica e biológica na melhoria dos atributos físicos de um Latossolo muito argiloso e no incremento do rendimento de soja. Revista Brasileira de Ciência do Solo, Viçosa, v. 32, n. 4, p. 1723-1734, 2008. Disponível em: 
$<$ http://www.scielo.br/pdf/rbcs/v32n4/a37v32n4.pd f>. Acesso em: 18 de novembro de 2013.

PIRES, L. F; ROSA, J. A; TIMM, L. C. Comparação de métodos de medida da densidade do solo. Acta Scientiarum. Agronomy, Maringá, v. 33, n. 1, p. 161-170, 2011. Disponível em: $<$ http://www.scielo.br/pdf/asagr/v33n1/v33n1a23.p df $>$. Acesso em: 18 de novembro de 2013.

RALISCH, R. et al. Avaliação em um solo argiloso sob plantio direto de uma escarificação na evolução da resistência do solo à penetração. In:

CONGRESSO BRASILEIRO DE ENGENHARIA AGRÍCOLA, 30., 2001, Foz do Iguaçu. Anais...

Foz do Iguaçu: Sociedade Brasileira de Engenharia Agrícola, 2001. 1 CD-ROM.

REICHARDT, K.; TIMM, L. C. Solo, planta e atmosfera: conceitos, processos e aplicações. Barueri: Manole, 2008.

REICHERT, J. M.; REINERT, D. J.; BRAIDA, J. A. Qualidade dos solos e sustentabilidade de sistemas agrícolas. Ciência \& Ambiente, Santa Maria, v. 27, n. 1, p. 29-48, 2003. Disponível em: $<$ http://www.fisicadosolo.ccr.ufsm.quoos.com.br/d ownloads/Producao_Artigos/5.pdf >. Acesso em: 18 de novembro de 2013.

SILVA, V. R.; REINERT, D. J.; REICHERT, J. M. Comparação entre métodos do cilindro e do torrão na determinação da porosidade e da densidade do solo. Ciência Rural, Santa Maria, v. 30, n. 6, p. 1065-1068, 2000. Disponível em: <http://www.scielo.br/pdf/cr/v30n6/a25v30n6.pdf>. Acesso em: 18 de novembro de 2013.

SILVEIRA, P. M. et al. Efeitos do manejo do solo sob plantio direto e de culturas na densidade e porosidade de um Latossolo. Bioscience Journal, Uberlândia, v. 24, n. 3, p. 53-59, 2008. Disponível em: < http://www.seer.ufu.br/index.php/biosciencejournal /article/view/6787/4481>. Acesso em: $18 \mathrm{de}$ novembro de 2013.

STONE, L. F.; SILVEIRA, P. M. Efeitos do sistema de preparo e da rotação de culturas na porosidade e densidade do solo. Revista Brasileira de Ciência do Solo, Viçosa, v. 25, n. 2, p. 395-401, 2001. Disponível em: $<$ http://sbcs.solos.ufv.br/solos/revistas/v25n2a15.pd f>. Acesso em: 18 de novembro de 2013.
TAVARES FILHO, J. et al. Resistência do solo à penetração e desenvolvimento do sistema radicular do milho (Zea mays) sob diferentes sistemas de manejo em um Latossolo Roxo. Revista Brasileira de Ciência do Solo, Viçosa, v. 25, n. 3, p. 725-730, 2001. Disponível em:

$<\mathrm{http}$ ://sbcs.solos.ufv.br/solos/revistas/v25n3a22.pd f>. Acesso em: 18 de novembro de 2013.

TORMENA, C. A. et al. Densidade, porosidade e resistência à penetração em Latossolo cultivado sob diferentes sistemas de preparo do solo. Scientia Agricola, Piracicaba, v. 59, n. 4, p.795-801, 2002. Disponível em: < http://www.scielo.br/pdf/sa/v59n4/a26v59n4.pdf > . Acesso em: 18 de novembro de 2013.

VAN REMORTEL, R. D.; SHIELDS, D. A. Comparison of clod and core methods for determination of soil bulk density.

Communications in Soil Science and Plant

Analysis, Philadelphia, v. 24, n. 6, p. 2517-2528, 1 